\title{
PFLEGE
}

\section{Aktionen allein helfen nicht}

VON MONA FROMMELT UND ROLAND SCHMIDT

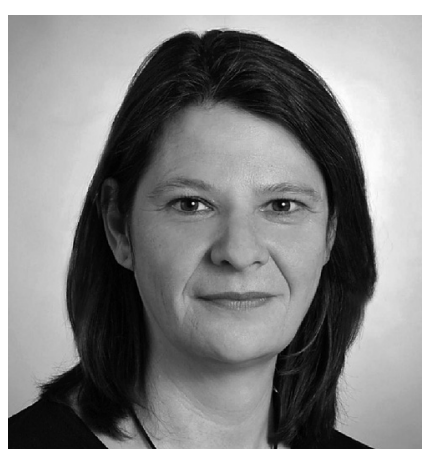

Mona Frommelt ist Ärztin und Direktorin der Hans-WeinbergerAkademie der Arbeiterwohlfahrt in Bayern.

www.hwa-online.de

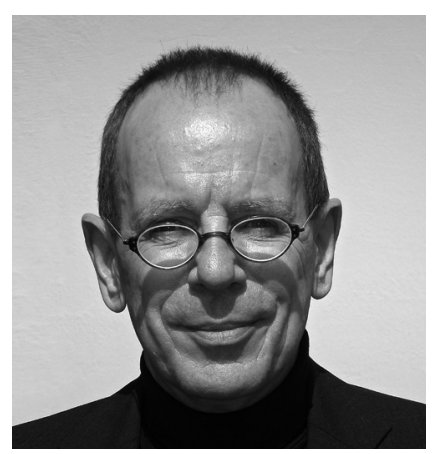

Prof. Dr. Roland Schmidt ist Hochschullehrer für Gerontologie und Versorgungsstrukturen an der Fachhochschule Erfurt. www.fh-erfurt.de/soz/so/ lehrende/prof-dr-roland-schmidt

\author{
Insbesondere die Pflege gilt als das Geschäftsfeld der \\ Sozialwirtschaft, das vom Mitarbeitermangel am stärksten \\ betroffen ist. Mit Teilstrategien wie der verstärkten \\ Gewinnung junger Menschen für das Berufsfeld und die \\ Anwerbung ausländischer Fachkräfte wird das Problem \\ jedoch nicht zu lösen sein. Vielmehr kommt es darauf an, \\ intelligente Formen der Prozessoptimierung innerhalb der \\ Pflege und in den Gesundheitsbereich hinein zu finden.
}

Modellrechnungen zum zukünftigen Bedarf an Pflegefachkräften rücken zumeist den Morbiditätsrückgang im hohen Alter sowie den Zusammenhang von Mortalität und Morbidität in den Fokus. Anstöße zur weiteren Profilierung von Disziplin und Profession, aber auch die Zunahme des Wettbewerbs zwischen Versorgungssektoren und Versorgungssystemen hingegen werden in solchen Betrachtungen zumeist ausgespart.

Unser Beitrag skizziert solche Entwicklungen, die bereits mit mehr oder weniger Breitenwirkung in Gang gesetzt sind. Sie zeigen eindrücklich, dass die Gestalt der Pflegeprofession und Versorgungsstrukturen im Kontext von Modellrechnungen keinesfalls konstant gesetzt werden dürfen. Der ungedeckte Bedarf an Fachkräften wird sich nicht in allen Segmenten pflegerischen Handelns gleichermaßen gravierend niederschlagen: Es wird attraktive und weniger attraktive Praxisfelder gegeben und es werden sich unterschiedliche Funktionen, Qualifikations- und Verantwortungsniveaus herauskristallisieren, die Karrieremöglichkeiten eröffnen.

Gestalt und Funktionen der Pflegeberufe befinden sich im Wandel. Dies resultiert aus versorgungsstrukturellen Impulsen im Bereich SGB V und SGB XI und folgt der Ausdifferenzierung von Qualifikationsniveaus und Kompetenzprofilen. In diesem sich zuletzt normativ fortlaufend neu speisenden Prozess ist auch die Debatte um fehlende Fachkräfte und über Strategien deren teilweiser Kompensation $\mathrm{zu}$ verorten.

\section{Vertikale Differenzierung}

Von »der Pflege« zu sprechen ist, das sei eingangs betont, eine letztlich wenig hilfreiche Reduktion der sich steigernden Komplexität dieses Berufsfeldes. Die Ausdifferenzierung beruflicher Aus-, Fort- und Weiterbildungen als Antwort auf die rasanten Entwicklungen des Bedarfs an Pflege, getrieben von vielschichtigen Interessen und Stakeholdern an und in diesem Feld, ist, verglichen mit dem ärztlichen Bereich, um einiges vielseitiger und gleichzeitig schwerer $\mathrm{zu}$ fassen, da diese deutlich weniger institutionell und strukturell gebündelt sind. So fehlen »der Pflege« im Gegensatz $\mathrm{zu}$ »der Medizin« vor allem kammerrechtlich und berufsrechtlich gesteuerte Fort- und Weiterbildungen. Aber auch die Ausbildungen, obwohl bundeseinheitlich gesetzlich geregelt (z. B. im Kranken- und Altenpflegegesetz), sind länderrechtlich äußerst heterogen ausgestaltet. Dies betrifft beispielsweise die strukturelle Regelung der Schul- und Ausbildungstypen und die Finanzierung der Ausbildung.

Diese Situation führt zum einen zur Ausprägung vielseitiger Ausbildungen 
und Assistenzberufe (1) und zum anderen $\mathrm{zu}$ vertikalen und horizontalen Ästen von Spezialisierungen und Spezifizierungen. Motive für die Einleitung solcher Diversifizierungsprozesse im Ausbildungsbereich der drei großen Kernberufe (Gesundheits- und Krankenpflege, Altenpflege und Kinderkrankenpflege) und in den spezifizierenden Qualifikationen (Leitungs- und Assistenzfunktionen) gründen zum einen in der Steigerung der Attraktivität des gesamten Berufsfeldes »Pflege « und zum anderen in dem Bemühen um Sicherung der Qualität der Pflege angesichts des anhaltenden und wachsenden Fachkräftemangels. Zwei große Trends prägen sich in dieser Gemengelage der Pflege jedoch zuletzt deutlicher aus:

- Zunächst ist die Unterscheidung zwischen Steuerungsverantwortung und Verrichtungsdurchführung anzusprechen (vgl. BSG-Urteil B 3 P 14/07 R vom 22.4.2009). Solche Differenzierungen in der Verantwortungsübernahme für die Steuerung des Pflegeprozesses werden beispielsweise in Rahmen des Primary Nursings (2), aber auch im Kontext von Case- und Care Management deutlich. Aufgaben und Verantwortung in den Bereichen Kommunikation, Beratung, Pflegediagnostik und Pflegeplanung sowie zur Steuerung und Auswertung der Durchführung von Pflege erfordern hohe soziale, integrative sowie Fach- und Methodenkompetenz (= qualitätssichernde Funktion). Mit der Entscheidung über den Hilfeumfang und die hierbei erforderlichen Qualifikationen der Verrichtungen übernehmenden Mitarbeiter stellt sich zugleich das Erfordernis eines fallbezogen rationalen Ressourceneinsatzes.

- Zudem ist hier die Fokussierung der Pflege auf pflegerische Bedarfslagen anzusprechen mit der Folge einer Delegation von Sozialfunktionen auf die Assistenzberufe. Dabei ist das Erfordernis zur Qualifikation solcher Assistenzfunktionen ist nicht immer allen verantwortlichen Akteuren aus Politik, der Leistungsträger und Leistungserbringer bewusst.

Eine Schärfung des Berufsprofils »Pflege " in der Kernkompetenz, also der Steuerung von inter- und intrasektoralen Prozessen, könnte in mehreren Dimensionen einen Beitrag zur Minimierung des Problems »Fachkräftemangel « leisten. Effekte, die hierdurch erwartet werden, reichen vom Imagegewinn des Berufsfeldes und der Prozessoptimierung über eine höhere Arbeitszufriedenheit (und damit auch: längere Verweildauer im
Beruf und weniger Überforderung mit Burn-out-Risiken dank klarerer Aufgabenverteilung und Delegation) bis hin zur Effizienz- und Qualitätssteigerung und nicht zuletzt zu potenziellen Kosteneinsparungseffekten.

Kurz: Es geht um die Frage der Rationalisierung der Pflege. Weiterhin ist in der vollstationären Pflege eine mittels Primary Nursing wahrgenommene Prozessverantwortung auch als Alternative zu einer bedarfsunabhängigen und daher überkommenen Fachkraftquote nutzbar zu machen.

\section{Heilkundebefähigung}

Mit Verabschiedung des Pflege-Weiterentwicklungsgesetzes hatte der Gesetzgeber mit $\mathbb{S} 63$ Abs. 3c SGB V einen spät aufgegriffenen - Impuls gesetzt, damit ein gemeinsamer Rahmen für Modelle zur Erprobung der Neuverteilung der Aufgaben im Gesundheitswesen auf der Grundlage einer bundesweit gültigen Richtlinie gemäß $\$ 63$ Abs. 3c SGB $\mathrm{V}$ (Heilkundeübertragungs-Richtlinie) entwickelt wird.

Die Verantwortung hierfür wurde dem Gemeinsamen Bundesausschuss (GBA) übertragen. (3) In der Richtlinie hat es der GBA vermieden, die Kontroverse »Delegation vs. Substitution « aufzugreifen. Vor allem die Ärztekammern

\section{Was versteht man eigentlich unter Care Management und Primary Nursing?}

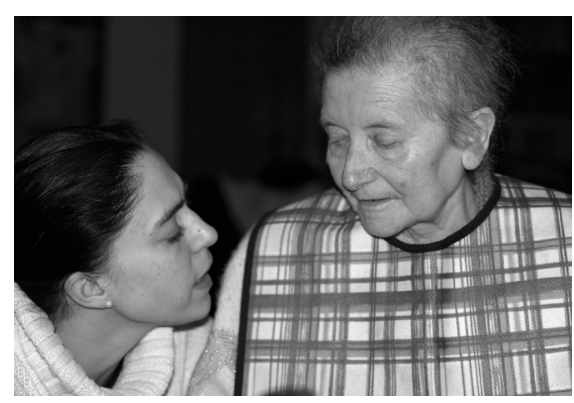

Als zwei Möglichkeiten einer intelligenteren Form der Prozesssteuerung und Prozessoptimierung im System Pflege gelten Care Management und Primary Nursing.

Care Management zielt darauf $a b, a b-$ gestimmte Versorgungsstrukturen zu implementieren, um insbesondere bei komplexen gesundheitlichen Problemen, die verbunden sind mit hohen Risiken für Betroffene und mit erheblichen Kosten für Leistungsträger, sektoren- und disziplinübergreifend Versorgungsabläufe zu rationalisieren. Dabei geht es zum einen um die Vermeidung von Unter-, Über- und Fehlversorgung durch eine systematisch aufeinander bezogene Diagnostik, Behandlung und Pflege sowie zum anderen um eine bessere Passung zwischen der professionellen Expertise, was evidenzund erfahrungsbasiert wirksame Interventionen im konkreten Fall sind, mit den individuellen Versorgungspräferenzen, die chronisch kranke Menschen mit Blick auf ihre Ressourcen/Potenziale und Beeinträchtigungen äußern. Erstgenanntes fokussiert den Effekt, letztgenanntes die subjektive Lebensqualität, also das Wohlbefinden. Integrierte Versorgungskonzepte kombinieren in einem jeweils besonderen Mischungsverhältnis Systemsteuerung (Care Management) mit Fallsteuerung (Case Management).

Primary Nursing (oft übersetzt mit Bezugspflege oder Primär-Pflege) ist ein in den 196oer Jahren entwickeltes Pflegesystem aus den USA. Es geht zurück auf Marie Monthey. Im Unterschied zur Funk- tionspflege oder Formen der Bereichspflege, wie z. B. der Gruppenpflege, übernimmt ausschließlich eine Pflegeperson die Verantwortung für die Aufnahme des Patienten, die Pflegeplanung und somit für den gesamten Pflegeprozess bis hin zur Entlassungsplanung. Die Aufgaben der Stationsleitung beschränken sich auf die Koordination und auf das Stationsmanagement. Primary Nursing ist gekennzeichnet durch vier Kernelemente: Übertragung und Übernahme individueller Verantwortung für pflegerische Entscheidungen durch eine Pflegende; Zuteilung der täglichen pflegerischen Arbeit nach der Fallmethode; direkte Kommunikation; Pflegeplanender ist zugleich Pflegedurchführender.

Quellen: www.fh-erfurt.de/soz/fileadmin/ SO/Dokumente/Lehrende/Schmidt Roland_Prof_Dr/Publikationen/Care $M a-$ nagement.pdf. www.pflegewiki.de/wiki/ Primary_Nursing. www.dbfk.de/Deutsches-Netzwerk-Primary-Nursing.php 
insistierten in der Diskussion des Richtlinienentwurfs und nach seiner Verabschiedung auf die Aufrechterhaltung der ärztlichen Diagnose als Grundlage jeglicher Tätigkeit im Bereich der Heilkunde.

Gleichwohl war und blieb im Entwurf enthalten, dass Pflegefachkräfte im Rahmen von Modellversuchen weitreichende Verantwortung übertragen bekommen können (wie z. B. selbstständig Verordnungen vorzunehmen). Dies hat die Kassenärztliche Bundesvereinigung (KBV) veranlasst, (erfolglos) zu intervenieren, weil man hier die Gefahr sieht, dass eine neue Gruppe von Leistungserbringern entsteht.

Die Modellversuche sind auf die Indikationen Bluthochdruck, Demenz, chronische Wunden sowie Diabetes Typ I und II beschränkt. Diagnose und Indikationsstellung bleiben in der Verantwortung des Arztes. Dies gilt auch für den Therapieplan. Modellvorhaben gemäß Heilkundeübertragungs-Richtlinie sind auf acht Jahre befristet und sie werden im Hinblick auf ihren Nutzen für Patienten evaluiert. Im Falle positiver Ergebnisse kann das Modell Bestandteil der Regelversorgung werden.

Diese ersten Impulse zu einer Neuordnung der Aufgabenverteilung zwischen den Gesundheitsprofessionen demonstrieren, dass sich die alten Grenzziehungen und Domänen allmählich lockern und neu justieren.

\section{Pflegerisches Fallmanagement}

Das Begriffsverständnis von Pflegeberatung ist vielschichtig und wird unterschiedlich gebraucht. Im Folgenden ist Pflegeberatung gemäß \ 7a SGB XI gefasst, eine Norm, die seit Januar 2009 jedem Bürger einen Anspruch auf kostenlose individuelle Pflegeberatung und - bei Bedarf - umfassende Hilfestellung zuerkennt.

Ziel war und ist es, wenn sich Information oder Beratung fallbezogen als nicht ausreichend erweisen, ein individuelles Fallmanagement in der Pflege zu installieren. Dieses weist aber zugleich über das im engeren Sinn Pflegerische hinaus, da im Kontext des Fallmanagements zudem die tägliche Lebensführung und die damit verbundenen bedeutsamen Bedarfe inter- und intrasektoral mitgedacht und gesteuert werden müssen. Damit ist Pflegeberatung rechtlich gesehen zunächst einmal als Instrument

der Versorgungssteuerung angelegt. In realiter wird dieses Szenario jedoch so heterogen und vielschichtig ausgestaltet, wie dies in einer föderalen, sektoralen und fragmentierten Landschaft gesundheitlicher Leistungserbringung nur möglich ist.

Vor und zu Beginn des damaligen Gesetzgebungsverfahrens wurde der Aufgabenbereich denn auch zutreffend als »Care Management " ausbuchstabiert, weil eingangs die Steuerung von Versorgungsprozessen im Fokus stand, die über die Steuerung der Einzelperson

\section{"Durch die Übertragung weitreichender Verantwortung an Pflegefachkräfte könnte eine neue Gruppe von Leistungserbringern entstehen"}

als »Fall« hinausreichte. Pflegeberatung sollte damit nicht nur der Orientierung des »Kunden« am Quasi-Markt dienen, sondern in einem zweiten Strang auf die Systemebene mittels Gestaltung von Pflege und der mit ihr verbundenen gesundheitlichen und sozialen Dienstleistungen einwirken. Diese Ausrichtung wurde im Gesetzgebungsverfahren und im Zuge der Kompromissfindung zwischen heterogenen Interessenlagen allerdings verunklart.

\section{Pflegeberatung}

Mit dem Einzug von Case Management und Care Management im Gewand der Pflegeberatung wurde ein Paradigmenwechsel mit Blick auf die Grundanlage von Pflege und die Gestaltung von Pflegeprozessen in allen Bereichen eingeläutet: von der ambulanten über die teilstationären hin zur stationären Pflege, vom Altenpflegeheim bis zum Krankenhaus sowie über die Bereiche Prävention, Kuration, Rehabilitation, Prävention und Palliation hinweg.

Grundlegend sind hier das Verständnis und der Zugang zum Pflegebedürftigen und seinen Angehörigen mittels Handeln nach Vereinbarungen, interessensbasierten Aushandlungsprozessen, Sorgeberatung und Nutzerorientierung, Hilfe zur Selbsthilfe (Selbstmanagement) sowie anwaltschaftlicher Unterstützung. $\mathrm{Zu}$ diesem Verständnis sind (a) die Pflegeplanung als Spezifizierung bzw. Teilsegment der Versorgungsplanung, (b) die ihr zugrundeliegende Sicht des Pflegebedürftigen und (c) die hierauf basierende Gestaltung von Pflegeprozess und Pflegehandeln anschlussfähig.

Spezifische Anwendung findet Caseund Care Management vor allem in der Pflegeüberleitung, im Entlassungsmanagement, in der Altenpflege als hochkomplexen Pflegekontext sowie im Alltagsmanagement (insbesondere in neuen Versorgungsformen an der Schnittstelle zwischen ambulant, teilstationär und stationär) und nicht zuletzt in der Pflegeberatung (durch Koordinierungsstellen, IAV-Stellen oder der von den Pflegekassen vorgehaltenen Pflegeberatung und in Pflegestützpunkten).

Von hoher Bedeutung ist, angesichts dieser Komplexität von Steuerungsansätzen, die Etablierung eines übergeordneten Rahmens, innerhalb dessen eine Governance-Kompetenz aufzubauen wäre. Diese ist angezeigt, damit

- die Vielfalt an Prozesssteuerungen und Beratungsansätzen im Sinne des Case- und Care Managements ausbalanciert werden kann,

- regionale Gegebenheiten Berücksichtigung finden,

- eine Vielzahl von gleich wirksamen und gleichwertigen Lösungen gefunden werden und

- das Recht der Bürger auf gesetzliche Gleichbehandlung gewährleistet ist.

\section{Resümee}

Herausforderung für die Zukunft der Pflege angesichts des nachhaltigen Problems des Fachkräftemangels wird sein, intelligente Formen der Prozesssteuerung und Prozessoptimierung innerhalb der Pflege und transsektoral in den Gesundheitsbereich hinein anzuwenden und diese in den Rahmen der Grundsätze einer Good Governance öffentlichen Handelns einzubinden. 
Hierbei sind die Prinzipien der Offenheit, der Partizipation, der Verantwortlichkeit und der Effektivität verpflichtend. (4) Die Lösung dieser Aufgabe ist der Ausgangspunkt sämtlicher weiterer Teilstrategien wie beispielsweise verstärkte Gewinnung junger Menschen für das Berufsfeld, Weiterqualifikation bereits in der Pflege befindlicher Mitarbeiterinnen, Anwerbung ausländischer Fachkräfte, Durchlässigkeit beruflicher und akademischer Bildung.

\section{Anmerkungen}

(1) Klie/Guerra: Synopse zu Service-, Assistenz- und Präsenzberufen in der Erziehung, Pflege und Betreuung (Care). Freiburg im Breisgau 2006.

(2) DBfK e. V./Deutsches Netzwerk Primary Nursing (Hg.): Personalentwicklung im Primary Nursing. Denkanstöße, Diskussionsgrundlagen und Impulse, o. O., Juli 2010. Primary Nursing ist eine Möglichkeit, Pflege zu organisieren. Dabei wird die umfassende Verantwortungsübernahme durch eine benannte Pflegefachkraft für die Pflege und Versorgung bestimmter Patienten in den Mittelpunkt gestellt.

(3) Gemeinsamer Bundesausschuss: Richtlinie des Gemeinsamen Bundesausschusses über die Festlegung ärztlicher Tätigkeiten zur Übertragung auf Berufsangehörige der Alten- und Krankenpflege zur selbstständigen Ausübung der Heilkunde im Rahmen von Modellvorhaben nach $\mathbb{S} 63$ Abs. 3c SGB V (Richtlinie nach $\mathbb{} 63$ Abs. 3c SGB V) vom 20. Oktober 2011.

(4) GKV-Spitzenverband (Hg.): Pflegeberatung. Berlin 2012 (Autoren: Thomas Klie, Mona Frommelt, Ulrich Schneekloth, Claus Heislbetz u. a.).

\title{
Handbuch Controlling
}

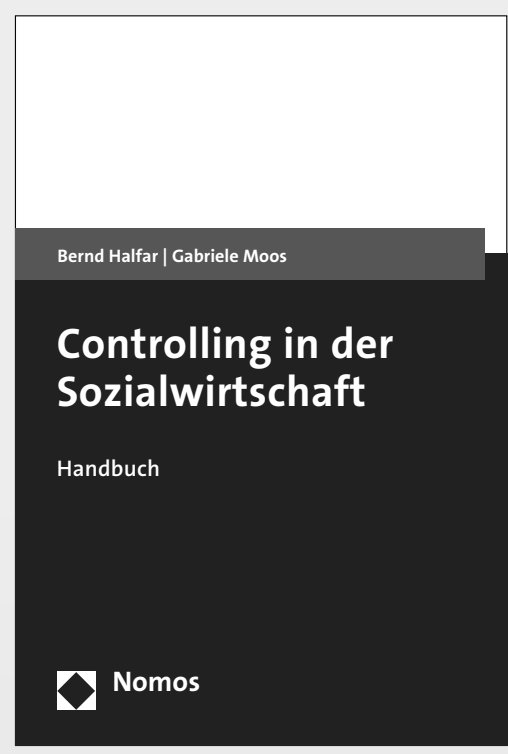

\author{
Controlling in der Sozialwirtschaft \\ Handbuch \\ Von Prof. Dr. Bernd Halfar und Prof. Dr. Gabriele Moos \\ 2013, ca. 300 S., brosch., ca. 34,- €, ISBN 978-3-8329-6327-9 \\ Erscheint ca. Juni 2013
}

Das von Bernd Halfar und Gabriele Moos vorgelegte Handbuch setzt an den Besonderheiten sozialer Dienstleistungsunternehmen an und entwickelt Vorschläge für ein praxistaugliches Controlling, das über das übliche Finanzcontrolling hinausreicht. Geschildert werden typische Fälle aus der Personaleinsatzplanung, aus dem Gebäudemanagement stationärer Einrichtungen, aus dem Qualitätsmanagement, aus der externen und internen Personalbeschaffung, aus dem Kostenmanagement, der Investitionsrechnung, der Verwaltungsorganisation, aus dem IT-Management, dem Facility Management und der Wirkungsmessung sozialer Dienstleistungen.

Der Leser findet thematisch geordnete Kennzahlenlisten und Anregungen, wie das Controlling die Produktivität sozialwirtschaftlicher Unternehmen analysieren kann.

Bestellen Sie jetzt telefonisch unter 07221/2104-37

Portofreie Buch-Bestellungen

unter www.nomos-shop.de/13315

Nomos 